COMMENT. Hashimoto encephalopathy (HE) is characterized by seizures, neurologic and psychiatric manifestations, and elevated titers of serum anti-thyroid antibodies. It is responsive to corticosteroids. Plasmapheresis is a novel method of acute treatment. HE should be considered, along with anti-N-methyl-D-aspartate-receptor, voltage-gated potassium channel antibody-associated limbic encephalitis, and herpes simplex virus encephalitis, in the differential diagnosis of a child with acute personality changes and seizures resistant to antiepileptic medication. The pathogenesis of HE is associated with high serum anti-thyroid antibody titers; thyroid hormone levels are usually normal or slightly low. An autoimmune disease process is likely.

\title{
POLG NOVEL MUTATION WITH ALPERS SYNDROME
}

Researchers at University Hospital, Berne, Switzerland describe the molecular genetic analysis of POLG in a 3.5 years old boy with VPA-induced fatal liver failure and encephalopathy (Alpers-Huttenlocher syndrome, AHS). Mutations in the POLG gene are a common cause of inherited mitochondrial disease in children and adults. They are involved with various neurodegenerative diseases, including Alpers syndrome, and result in accumulation of multiple mtDNA deletions and/or depletions of mtDNA in muscle, brain and liver. Some POLG mutations lead to a range of clinical phenotypes that predispose to fatal liver failure after exposure to VPA. POLG analysis in mitochondrial diseases helps in confirmation of AHS and optimizes clinical management. (Schaller A, Hahn D, Jackson CB, et al. Molecular and biochemical characterization of a novel mutation in POLG associated with Alpers syndrome. BMC Neurology 2011;11:4-11). (Respond: Dr Andre Schaller. E-mail: andre.schaller@insel.ch).

COMMENT. The study extends the list of $P O L G$ mutations associated with VPA hepatoxicity. A report of reversible valproate hepatotoxicity due to mutations in mitochondrial DNA polymerase gamma (POLG1) is cited. (McFarland R et al. Arch Dis Child 2008;93(2):151-153).

\section{DEMYELINATING DISEASE}

\section{HEAD AND BRAIN SIZE IN PEDIATRIC MULTIPLE SCLEROSIS}

Researchers at the Montreal Neurological Institute, the Hospital for Sick Children, Toronto, Canada; and Department of Neurology, Rennes, France conducted MRI measurements of whole brain and regional white matter, gray matter, and deep gray matter structure volumes in 38 patients (mean age 15.2+/-2.4 years) with pediatric-onset relapsing-remitting multiple sclerosis (MS). Mean age at MS onset was 12.1 years; mean disease duration 3.1 years. Values obtained from sex-matched healthy controls enrolled in the MRI Study of Normal Brain Development were used as controls. The intracranial volume and normalized brain volume $\mathrm{z}$ scores were significantly lower in patients with MS compared with controls. Thalamic volumes in MS patients were lower even after correction for global brain volume decreases. Reduced thalamic and brain volumes 
correlate moderately with increased disease duration. Head size of pediatric MS patients is lower than controls. (Kerbrat A, Aubert-Broche B, Fonov V, et al. Reduced head and brain size for age and disproportionately smaller thalami in child-onset MS. Neurology January 17, 2012;78(3);194-201). (Response and Reprints: Dr Collins, E-mail: louis.collins@mcgill.ca).

COMMENT. Onset of MS during childhood is associated with smaller head size, brain volume, and even smaller thalamic volume. Cognitive impairment is a notable feature of pediatric MS. (Amato M et al. Neurology 2007;70:1891-1897), and thalamic volume correlates with cognitive performance of children with MS. (Till C et al. Neuropsychology 2011;25;319-332).

\section{HEADACHE DISORDERS}

\section{SHARED GENETIC ETIOLOGY FOR MIGRAINE AND EPILEPSY}

Shared loci for migraine and epilepsy were found on chromosomes 14q12-q23 and 12q24.2-q24.3 in a linkage analysis study of a Finish family with a complex phenotype, in a report from Folkhalsan Institute and other centers in Helsinki and Oulu, Finland; University of California, Los Angeles; and Wellcome Trust Sanger Institute, Cambridge, UK. Of 60 family members, $12(20 \%)$ had idiopathic epileptic seizures, and 8 of the $12(67 \%)$ also had migraine. Seven (12\%) had febrile seizures. (The novel migraine locus identified on chromosome 12 has previously been linked to febrile seizures [Gurnett CA et al. Neurogenetics 2007;8:61-63]). Ten family members (17\%) had sudden somnolence lasting a few minutes to 2 hours and associated with centrotemporal EEG abnormalities. Thirty-three of the 60 family members $(55 \%)$ had migraine (20 [33\%] without and $13[22 \%]$ with aura), and $37(62 \%)$ had either migraine or epilepsy. Nine (15\%) family members had both migraine and epilepsy. (Polvi A, Siren A, Kallela M, et al. Shared loci for migraine and epilepsy on chromosome 14q12-q23 and 12q24.2-q24.3. Neurology January 17, 2012;78:202-209). (Response and Reprints: Dr Polvi. E-mail: anne.polvi@helsinki.fi).

COMMENT. Migraine and epilepsy share a common genetic etiology. Of patients with migraine, $6 \%$ have epilepsy and up to $26 \%$ of patients with epilepsy have migraine (Ottman R, Lipton RB. Neurology 1994;44:2105-2110 and others, cited by authors). Antiepileptic medications are effective in the prophylaxis of migraine. (Barbanti P et al. Migraine prophylaxis: what is new and what we need? Neurol Sci 2011;32(suppl 1):S111-S115).

\section{EFFECT OF HEADACHE ON ACADEMIC PERFORMANCE}

Researchers at University of Pernambuco, Recife, Brazil interviewed 344 randomly selected, university, social communication students to determine the 1-year prevalence of headache, types of headache, and the effects on academic performance. The mean age was 23.4 years; $57.3 \%$ were women. Headache prevalence was $87.2 \%$ 